PROCEEDINGS OF THE

AMERICAN MATHEMATICAL SOCIETY

Volume 134, Number 12, December 2006, Pages 3449-3456

S 0002-9939(06)08589-3

Article electronically published on June 27, 2006

\title{
ON WEYL GROUP EQUIVARIANT MAPS
}

\author{
ADAM KORÁNYI AND RÓBERT SZŐKE
}

(Communicated by Dan M. Barbasch)

\begin{abstract}
We prove an equivariant analogue of Chevalley's isomorphism theorem for polynomial, $C^{\infty}$ or $C^{\omega}$ maps.
\end{abstract}

\section{INTRODUCTION}

In the theory of symmetric spaces a fundamental role is played by Chevalley's extension theorem [H2, pp. 299, 340], [HC]:

Suppose $\mathfrak{g}$ is a semisimple Lie algebra of noncompact type over $\mathbb{R}, \theta$ a Cartan involution, $\mathfrak{g}=\mathfrak{k}+\mathfrak{p}$ the corresponding Cartan decomposition of $\mathfrak{g}$, and $\mathfrak{a} \subset \mathfrak{p}$ a maximal Abelian subspace. Let $G$ be a connected Lie group with Lie algebra $\mathfrak{g}$, and let $K$ be the analytic subgroup corresponding to $\mathfrak{k}$. Then $K$ acts on $\mathfrak{p}$ by the adjoint representation and $W$, the Weyl group, acts on $\mathfrak{a}$. The theorem states that every $W$-invariant polynomial on $\mathfrak{a}$ extends to a unique $K$-invariant polynomial on $\mathfrak{p}$. It is an immediate consequence that the two polynomial algebras in question are isomorphic.

This theorem remains true if "polynomial" is replaced by $C^{\infty}$ or $C^{\omega}$ (see $\mathrm{D}$, [H2, p. 295], and the comments at the beginning of our Section 1).

It is natural to ask whether analogous results hold for $W$-equivariant polynomial (resp. $C^{\infty}, C^{\omega}$ ) mappings. In this paper we show that the answer is positive, and in fact a substantial part of the solution is already contained, somewhat indirectly, in [S] and [M1, M2].

Theorem 0.1. Any $W$-equivariant polynomial (resp. $\left.C^{\infty}, C^{\omega}\right)$ map $\mathfrak{a} \rightarrow \mathfrak{a}$ can be extended to a $K$-equivariant polynomial $\left(r e s p . C^{\infty}, C^{\omega}\right)$ map $\mathfrak{p} \rightarrow \mathfrak{p}$. The extension is unique.

The need for such an extension result arose in $[\mathrm{D}-\mathrm{Sz}]$ in constructing hyperkähler metrics on the tangent bundle of compact Hermitian symmetric spaces and where, for the particular map in question it was checked case-by-case for the classical groups. Another immediate consequence of the extension theorem is the following.

Received by the editors July 24, 2004 and, in revised form, July 4, 2005.

2000 Mathematics Subject Classification. Primary 20F55, 22E46, 53C35.

Key words and phrases. Symmetric spaces, equivariant maps, reflection groups.

The first author was partially supported by the National Science Foundation of the USA and by a PSC-CUNY grant.

The second author's research was partially supported by the Hungarian Science Foundation (OTKA) under grant T49449.

(C)2006 American Mathematical Society Reverts to public domain 28 years from publication 
Corollary 0.2. Let $(M, g)$ be a symmetric space of compact or noncompact type, $m_{0} \in M, \mathfrak{a} \subset T_{m_{0}} M$ a maximal flat subspace and $W$ the Weyl group. Then every $W$-equivariant $C^{\infty}$ (resp. $C^{\omega}$ ) map $\varphi$ from $\mathfrak{a}$ to $\mathfrak{a}$ extends uniquely to an isometry group equivariant $C^{\infty}$ (resp. $C^{\omega}$ ) map $\Phi$ from $T M$ to $T M$. $\Phi$ is a $\left(C^{\infty}\right.$ or $C^{\omega}$ ) diffeomorphism iff $\varphi$ is.

Theorem 0.1 provokes the next natural question. Does this theorem describe all possible $K$-equivariant maps? This is answered by our next result, Theorem 0.3.

We call a $K$-equivariant map $F: \mathfrak{p} \rightarrow \mathfrak{p}$ radial if there exists a maximal Abelian subspace $\mathfrak{a}$ in $\mathfrak{p}$ that is mapped into itself by $F$. Since $K$ acts transitively on the set of such a's, a radial map necessarily maps every maximal Abelian subspace of $\mathfrak{p}$ into itself.

Assume now that $\mathfrak{g}$ is simple. We say that $\mathfrak{g}$ is of Hermitian type if $\mathfrak{p}$ has a $K$-invariant complex structure, i.e., if the associated symmetric space is Hermitian symmetric.

Theorem 0.3. Let $F: \mathfrak{p} \rightarrow \mathfrak{p}$ be a K-equivariant polynomial (resp $C^{\infty}$ or $C^{\omega}$ ) map. If $\mathfrak{g}$ is not of Hermitian type, then $F$ is radial. Let $\mathfrak{g}$ be of Hermitian type and let $I$ be the complex structure on $\mathfrak{p}$. If $F_{j}: \mathfrak{p} \rightarrow \mathfrak{p}, j=1,2$, are arbitrary $K$-equivariant radial polynomial (resp. $C^{\infty}$ or $C^{\omega}$ ) maps, then $F=F_{1}+I F_{2}$ is a $K$-equivariant polynomial (resp. $C^{\infty}$ or $C^{\omega}$ ) map. Every $K$-equivariant polynomial (resp. $C^{\infty}$ or $\left.C^{\omega}\right)$ map $\mathfrak{p} \rightarrow \mathfrak{p}$ arises in this way.

In Section 1 we discuss a structure theorem for $W$-invariant $C^{\infty}$ or $C^{\omega} p$-forms inspired by Solomon's similar result for $W$-invariant polynomial $p$-forms (cf. $[\underline{\mathrm{S}}$, [H2, p. 363], [M1]). This structure theorem is important for our purpose because $W$-invariant one forms are essentially the same thing as $W$-equivariant maps (see Proposition 2.1).

In the first version of this paper we gave a complete proof of this theorem. But while our paper was refereed, we discovered the papers [M1, M2], where P. Michor already proved the result, essentially by the same method. Therefore, in this new version we only include details that are important for our purposes.

Theorem 0.1 is proved in Section 2, and Theorem 0.3 is proved in Section 3.

\section{1. $W$-INVARIANT $p$-FORMS}

Let $E$ be an $n$-dimensional real vector space and $W$ a finite reflection group on E. A theorem of Chevalley ( $[\mathrm{C}],[\mathrm{H} 2$, Theorem 3.1, p. 356]) says that there exist algebraically independent $W$-invariant real polynomials $j_{1}, \ldots, j_{n}$, such that every $W$-invariant real polynomial on $E$ is a polynomial of $j_{1}, \ldots, j_{n}$. In other words, setting $\mathcal{J}(x)=\left(j_{1}(x), \ldots, j_{n}(x)\right)$, for every $W$-invariant polynomial $f$ on $E$ we have $f=\bar{f} \circ \mathcal{J}$ with some polynomial $\bar{f}$ on $\mathbb{R}^{n}$. The same statement is true when $f$ (and $\bar{f}$ ) are in $C^{\infty} \underline{\mathrm{Sch}}, \underline{\mathrm{D}}$, or in $C^{\omega} \underline{\mathrm{L}}$. Note that these results immediately imply the $C^{\infty}$ and $C^{\omega}$ analogues of the Chevalley extension theorem.

The above results describe the structure of the $W$-invariant polynomial (resp. $C^{\infty}, C^{\omega}$ ) functions, i.e., $W$-invariant 0 -forms. We proceed to describe an analogous structure theorem for $W$-invariant $p$-forms, where $p>0$.

Proposition 1.1. Let $0 \in G \subset \mathbb{R}^{n-1}$ be open, $B=G \times(-\varepsilon, \varepsilon) \subset \mathbb{R}^{n}, f \in C^{k}(B)$ (resp. $C^{\infty}(B)$ or $\left.C^{\omega}(B)\right)$ and $f\left(x^{\prime}, 0\right) \equiv 0, x^{\prime} \in G$. Then $F:=\frac{f}{x_{n}} \in C^{k-1}(B)$ (resp. $C^{\infty}(B)$ or $\left.C^{\omega}(B)\right)$ and $F\left(x^{\prime}, 0\right)=\partial_{x_{n}} f\left(x^{\prime}, 0\right)$. 
Proof. Let $x=\left(x^{\prime}, x_{n}\right)$ be fixed and let $g: B \times[0,1] \rightarrow \mathbb{R}$ be defined as $g\left(x^{\prime}, x_{n}, t\right)=$ $f\left(x^{\prime}, t x_{n}\right)$. The Newton-Leibniz formula applied to the function $g(x,$.$) yields$

$$
f(x)=\int_{0}^{1}\left(\partial_{t} g\right)(x, s) d s=\int_{0}^{1} x_{n} \partial_{x_{n}} f\left(x^{\prime}, s x_{n}\right) d s .
$$

Proposition 1.2. Let $X$ be a $C^{\infty}$-manifold and $h \in C^{\infty}(X, \mathbb{R})$. Let $Z(h)$ denote the zeroes of $h$. Suppose $d h(x) \neq 0$ if $x \in Z(h)$. Now let $f \in C^{\infty}(X, \mathbb{R}),\left.f\right|_{Z(h)} \equiv 0$. Then $F:=\frac{f}{h} \in C^{\infty}(X, \mathbb{R})$. If $X, h, f \in C^{\omega}$, then $F \in C^{\omega}$ as well.

Proof. The implicit function theorem and Proposition 1.1 yield the statement.

Proposition 1.3. Let $X$ be a $C^{\omega}$-manifold and $h_{1}, \ldots, h_{r} \in C^{\omega}(X, \mathbb{R})$. Let $Z_{j}$ be the zero set of $h_{j}$ and $Z=\bigcup_{j=1}^{r} Z_{j}$. Assume that for each $j,\left.d h_{j}\right|_{Z_{j}} \neq 0$. Furthermore, suppose that for every component $M$ of $Z_{j}$, for each $k \neq j,\left.h_{k}\right|_{M} \not \equiv 0$. Let $f \in C^{\infty}(X, \mathbb{R})\left(\right.$ resp. $\left.\in C^{\omega}(X, \mathbb{R})\right)$ and $\left.f\right|_{Z} \equiv 0$. Then

$$
F=\frac{f}{h_{1} h_{2} \ldots h_{r}} \in C^{\infty}(X, \mathbb{R}) \quad\left(\text { resp. } \in C^{\omega}(X, \mathbb{R})\right) .
$$

Proof. We prove the statement by induction on $r$. For $r=1$ this is Proposition 1.2. Suppose we proved the statement for $r-1$. Then

$$
F_{r-1}=\frac{f}{h_{1} h_{2} \ldots h_{r-1}} \in C^{\infty}(X, \mathbb{R}) \quad\left(\text { resp. } \in C^{\omega}(X, \mathbb{R})\right) .
$$

Let $M$ be an arbitrary component of $Z_{r}$. Then for $1 \leq j \leq r-1,\left.h_{j}\right|_{M}$ is $\not \equiv 0$ and real-analytic. Therefore, the interior of $Z_{j} \cap M$ in $M$ is empty. Consequently, the set

$$
H:=M \backslash\left(\bigcup_{j=1}^{r-1}\left(Z_{j} \cap M\right)\right)
$$

is open and dense in $M$. By our assumption $\left.F_{r-1}\right|_{H} \equiv 0$. Hence $\left.F_{r-1}\right|_{M} \equiv 0$, yielding that $\left.F_{r-1}\right|_{Z_{r}} \equiv 0$. This together with Proposition 1.2 proves our claim.

Now let $E, W, j_{1}, \ldots, j_{n}$ be as at the beginning of this section.

Structure Theorem I. Let $\alpha$ be a $W$-invariant polynomial, $C^{\infty}$ or $C^{\omega} p$-form $(p>0)$ on $E$. Then $\alpha$ can be expressed uniquely as

$$
\alpha=\sum_{i_{1}<\cdots<i_{p}} \alpha_{i_{1}, \ldots, i_{p}} d j_{i_{1}} \wedge \cdots \wedge d j_{i_{p}},
$$

where $\alpha_{i_{1}, \ldots, i_{p}}$ are $W$-invariant polynomial, $C^{\infty}$ or $C^{\omega}$ functions on $E$.

The polynomial case is Solomon's theorem $\left[\mathbf{S}\right.$. The $C^{\infty}$ case was proved by P. Michor [M1, Lemma 3.3]. His proof could be slightly simplified by quoting [H2, Lemma 3.7, p. 361], that immediately yields formula (4) in [M1, p. 1636]. The real-analytic version follows the same line of reasoning as the $C^{\infty}$ case. To be more precise, let $\sigma_{1}, \ldots, \sigma_{r}$ be the reflections in $W$ and $\beta_{1}=0, \ldots, \beta_{r}=0$ the corresponding reflecting hyperplanes. Let $\pi=\prod_{i=1}^{r} \beta_{i}$. If a $C^{\omega}$ function $g$ satisfies $g \circ \sigma=\operatorname{det} \sigma^{-1} g$ for each $\sigma \in W$, then $g$ must vanish on the zero locus of $\pi$ and Proposition 1.3 implies that $g=\pi h$, where $h$ is a $W$-invariant $C^{\omega}$ function.

The rest of the proof of [M1, Lemma 3.3] goes through literally, $C^{\infty}$ replaced by $C^{\omega}$ everywhere, proving the real-analytic version. 
Remarks. 1) If $D$ is a $W$ invariant open subset of $E$ and $\alpha$ is a $W$-invariant $p$-form over $D$, the same proof shows that $\alpha$ can be expressed by the same formula as in the theorem, except the functions $\alpha_{i_{1}, \ldots, i_{p}}$ are defined only on $D$ and their smoothness (i.e., $C^{\infty}$ or real-analytic) agrees with that of $\alpha$.

2) The structure theorem can be restated as follows: For every $W$-invariant polynomial, $C^{\infty}$ or $C^{\omega} p$-form $\alpha$ on $E$ there exists a $p$-form $\bar{\alpha}$ of the same smoothness on $\mathbb{R}^{n}$ such that $\alpha=\mathcal{J}^{*} \bar{\alpha}$.

\section{EQUIVARIANT MAPS}

Let $E$ be a finite-dimensional real vector space. As usual we identify the tangent space of $E$ at all points with $E$. Let $b$ be a nondegenerate symmetric bilinear form on $E$. Given a 1-form $\alpha$ on $E$ (i.e., a section of $T^{*} E$ ) we associate to it the map $h_{\alpha}: E \rightarrow E$ defined by

$$
b\left(h_{\alpha}(p), v\right)=\alpha_{p}(v) \quad(\forall v \in E) .
$$

Clearly, $\alpha \rightarrow h_{\alpha}$ is a bijection between 1-forms on $E$ and maps of $E$ to $E$.

If $A$ is a linear transformation on $E$, we have $A_{*} v=A v$ under our identifications. If $A$ is orthogonal with respect to $b$, then

$$
\left(A^{*} \alpha\right)_{p}(v)=\alpha_{A p}(A v)=b\left(h_{\alpha}(A p), A v\right)=b\left(A^{-1} h_{\alpha}(A p), v\right) .
$$

Comparison with (2.1) shows that

$$
A^{*} \alpha=\alpha \Longleftrightarrow A^{-1} \circ h_{\alpha} \circ A=h_{\alpha},
$$

i.e., $\alpha$ is $A$-invariant iff $h_{\alpha}$ is $A$-equivariant.

So we have proved the following:

Proposition 2.1. Let $E$ be a finite-dimensional real vector space equipped with a nondegenerate symmetric bilinear form $b$. Let $G$ be a group acting on $E$ by $b$ orthogonal transformations and let $\alpha$ be a 1-form on $E$. Then $\alpha$ is $G$-invariant iff the corresponding map $h_{\alpha}: E \rightarrow E$ is G-equivariant.

Now let $f: E \rightarrow \mathbb{R}$ be a smooth function and $A$ a linear transformation on $E$. Since the pull-back by a smooth map commutes with the exterior derivative and the origin is a fixed point of $A$ we have

$$
f \circ A \equiv f \quad \text { iff } \quad A^{*} d f \equiv d f .
$$

We write $\nabla f$ for $h_{d f}$. This is then just the classical notion of the gradient of $f$ regarded as an $E$-valued function on $E$. Now (2.2), (2.3) and (2.4) together imply:

Proposition 2.2. Let $E$ be a finite-dimensional real vector space equipped with a nondegenerate symmetric bilinear form $b$. Let $G$ be a group acting on $E$ by $b$ orthogonal transformations and $f: E \rightarrow \mathbb{R}$ a differentiable function. Then the following statements are equivalent:

(1) $f$ is G-invariant,

(2) $d f$ is $G$-invariant,

(3) $\nabla f$ is G-equivariant.

Now let $j_{1} \ldots j_{n}$ be as at the beginning of Section 1. In light of Proposition 2.2, the maps $\nabla j_{i}: \mathfrak{a} \rightarrow \mathfrak{a}$ are $W$-equivariant. Furthermore, as a consequence of the Structure Theorem of Section 1 and of Proposition 2.1 we get the following. 
Proposition 2.3. Let $E^{n}$ be an $n$-dimensional real vector space and $W$ a finite reflection group on $E$. Then every $W$-equivariant polynomial (resp. $C^{\infty}$ or $C^{\omega}$ ) $\operatorname{map} \varphi: E \rightarrow E$ is of the form

$$
\varphi=\sum_{i=1}^{n} h_{i} \nabla j_{i},
$$

where each $h_{i}$ is a $W$-invariant polynomial (resp. $C^{\infty}$ or $C^{\omega}$ ) function.

We now return to the case of a real semisimple Lie algebra $\mathfrak{g}$ with $\mathfrak{k}, \mathfrak{p}, \mathfrak{a}, G, K$, $W$, as in the Introduction.

Proof of Theorem 0.1 . Let $\tilde{j}_{i}$ be the $K$-invariant extension of $j_{i}$ to $\mathfrak{p}$. In view of Proposition 2.3 and of the Chevalley extension theorem (and its $C^{\infty}$ and $C^{\omega}$ versions, cf. the beginning of Section 1) it suffices to show that each $\nabla j_{i}$ extends to a $K$-equivariant polynomial map $\mathfrak{p} \rightarrow \mathfrak{p}$. To prove this, we note that for $H \in \mathfrak{a}$ the $K$-orbit $O_{H}$ of $H$ is orthogonal to a, since every tangent vector at $H$ to $O_{H}$ is of the form $[Z, H]$ with $Z \in \mathfrak{k}$, and for every $H^{\prime} \in \mathfrak{a}, B\left([Z, H], H^{\prime}\right)=B\left(Z,\left[H, H^{\prime}\right]\right)=0$ (where $B$ is the Killing form). If $H$ is a regular element, comparison of the Iwasawa and Bruhat decompositions shows that the codimension of $O_{H}$ in $\mathfrak{p}$ equals dim a. Since $\tilde{j}_{i}$ is constant on $O_{H}$, it follows that

$$
\nabla_{\mathfrak{p}} \tilde{j}_{i}(H)=\left(\nabla_{\mathfrak{a}} j_{i}\right)(H) .
$$

By continuity this is then true for all $H \in \mathfrak{a}$. By Proposition $2.2, \nabla_{\mathfrak{p}} \tilde{j}_{i}$ is $K$ equivariant, so the proof is finished.

Recall from the Introduction that a $K$-equivariant map $F: \mathfrak{p} \rightarrow \mathfrak{p}$ is called radial, if there exists a maximal Abelian subspace $\mathfrak{a}$ in $\mathfrak{p}$, that is carried into itself by $F$. Theorem 0.1 yields an isomorphism between the space of $W$-equivariant polynomial, $C^{\infty}$, resp. $C^{\omega}$, maps and $K$-equivariant radial polynomial, $C^{\infty}$, resp. $C^{\omega}$, maps. The question whether there are other kinds of $K$-equivariant maps, will be addressed in the next section.

A differential form $\alpha$ on $\mathfrak{p}$ is called horizontal if $\alpha$ vanishes on the tangent vectors of the $K$-orbits, i.e., $\iota_{X^{\sharp}} \alpha=0$ for all $X \in \mathfrak{k}$, where $X^{\sharp}$ denotes the induced vector field on $\mathfrak{p}$. Clearly, a $K$-invariant 1 -form is horizontal iff the corresponding $K$ equivariant map is radial.

As in the proof of Theorem 0.1 , let $\tilde{j}_{i}$ be the $K$-invariant extension of $j_{i}$ to $\mathfrak{p}$.

Structure Theorem I, Proposition 2.2, Chevalley's extension theorem and its $C^{\infty}$ and $C^{\omega}$ versions imply:

Structure Theorem II. A polynomial (resp, $C^{\infty}$ or $C^{\omega}$ ) horizontal $p$-form on $\mathfrak{p}$ is $K$-invariant iff $\alpha$ can be expressed as

$$
\alpha=\sum_{i_{1}<\cdots<i_{p}} \alpha_{i_{1}, \ldots, i_{p}} d \tilde{j}_{i_{1}} \wedge \cdots \wedge d \tilde{j}_{i_{p}}
$$

where $\alpha_{i_{1}, \ldots, i_{p}}$ are $K$-invariant polynomials (resp. $C^{\infty}$, or $C^{\omega}$ functions). The imbedding $\iota: \mathfrak{a} \rightarrow \mathfrak{p}$ yields an isomorphism between the space of horizontal $K$ invariant $p$-forms on $\mathfrak{p}$ and the space of $W$-invariant $p$-forms on $\mathfrak{a}$ (cf. [M1, 3.7 Theorem]). 


\section{Proof of Theorem 0.3}

Let $\mathfrak{g}$ be a real simple Lie algebra of noncompact type, with $\theta, \mathfrak{k}, \mathfrak{p}, \mathfrak{a}, G, K$, $W$, as in the Introduction. As usual, we write $M, M^{\prime}$ for the centralizer, resp. the normalizer, of $\mathfrak{a}$ in $K$. We set

$$
p^{M}=\{v \in \mathfrak{p} \mid \operatorname{Ad}(k) v=v, \forall k \in M\} .
$$

Lemma 3.1. If $\mathfrak{g}$ is not of Hermitian type, then

$$
p^{M}=\mathfrak{a} .
$$

If $\mathfrak{g}$ is of Hermitian type, then

$$
p^{M}=\mathfrak{a} \oplus I \mathfrak{a} .
$$

Proof. Denote by $\Sigma$ the set of nonzero restricted roots with respect to $\theta$, a. Let $\mathfrak{g}_{\lambda}$ be the root space corresponding to $\lambda \in \Sigma$. The group $M$ maps every root space $\mathfrak{g}_{\lambda}$ into itself. Let $S$ denote the (possibly empty) set of all roots $\lambda \in \Sigma$ such that $M$ acts trivially on $\mathfrak{g}_{\lambda} . M^{\prime}$ (and so the Weyl group as well) acts on $\Sigma$ and it is not hard to see that $S$ is the union of full $W$ orbits. The $M$ action and $\theta$ commute on $\mathfrak{g}$ and $\theta \mathfrak{g}_{\lambda}=\mathfrak{g}_{-\lambda}$. This shows that $\lambda \in S$ iff $-\lambda \in S$.

Choose an ordering in the dual of $\mathfrak{a}$. Then

$$
\mathfrak{p}=\mathfrak{a} \oplus(I d-\theta)\left(\bigoplus_{\lambda>0} \mathfrak{g}_{\lambda}\right) .
$$

Denote by $S^{+}$the positive roots in $S$. (3.3) implies

$$
p^{M}=\mathfrak{a} \oplus(I d-\theta)\left(\bigoplus_{\lambda \in S^{+}} \mathfrak{g}_{\lambda}\right) .
$$

It is well known that in case $\operatorname{dim} \mathfrak{g}_{\lambda}>1, M$ acts transitively on the unit sphere in $\mathfrak{g}_{\lambda}$. Therefore, all root spaces in (3.4) are 1-dimensional.

Let $B$ be the Killing form of $\mathfrak{g}$ (which is positive definite on $\mathfrak{p}$ ) and for a $\lambda \in \Sigma$, denote by $A_{\lambda} \in \mathfrak{a}$ the vector such that $\lambda()=.B\left(., A_{\lambda}\right)$. Let

$$
A_{\lambda}^{\prime}:=\frac{2}{\lambda\left(A_{\lambda}\right)} A_{\lambda}
$$

Our statements involve only the adjoint action of $G$ and $K$. This is the same for any connected version of $G$. Therefore, in the following we may assume that $G$ is contained in the simply connected version of its complexification. [H1, (4), p. $322]$ then says, that $m_{\lambda}:=\exp _{G_{\mathbb{C}}}\left(i \pi A_{\lambda}^{\prime}\right) \in K$ and then obviously $m_{\lambda} \in M$ for each $\lambda \in \Sigma$.

Let $\lambda, \alpha \in \Sigma$ be arbitrary simple roots. Then

$$
\alpha\left(A_{\lambda}^{\prime}\right)=\frac{2 \alpha\left(A_{\lambda}\right)}{\lambda\left(A_{\lambda}\right)}=n(\alpha, \lambda)
$$

is the corresponding Cartan integer. Let $X_{\alpha} \in \mathfrak{g}_{\alpha}$ be a nonzero vector. Then

$$
A d\left(m_{\lambda}\right) X_{\alpha}=\operatorname{Ad}\left(\exp _{G_{\mathbb{C}}}\left(i \pi A_{\lambda}^{\prime}\right)\right) X_{\alpha}=e^{i \pi \alpha\left(A_{\lambda}^{\prime}\right)} X_{\alpha}=e^{i \pi n(\alpha, \lambda)} X_{\alpha} .
$$

(3.5) implies that $M$ will certainly be nontrivial on $\mathfrak{g}_{\alpha}$ (i.e., $\alpha \notin S$ ) if there is a simple $\lambda$ such that $n(\alpha, \lambda)$ is odd. This is the case if in the Dynkin diagram $\alpha$ is tied to some $\lambda$ by a single or a triple tie and also if there is a $\lambda$ tied to $\alpha$ by a double tie but $\alpha$ is shorter than $\lambda$ (in which case $n(\alpha, \lambda)=-1$ ). 
Assume now that $\Sigma$ is reduced. The discussion above shows that $S$ cannot contain any simple root, except possibly in the case $C_{l}(l \geq 2$, where $l=\operatorname{dim} \mathfrak{a})$, when there is one simple root, namely the longest one, to be called $\alpha$, with only a double tie to a shorter root, and in the case $A_{1}$, where we call $\alpha$ the only simple root.

One of the standard properties of reduced root systems (cf. [B, p. 279]) is that every $W$-orbit in $\Sigma$ contains a simple root. Therefore, $S$ may possible be nonempty only if the root system is of type $A_{1}$ or $C_{l},(l \geq 2)$ and $\alpha$ has multiplicity one. The table in [H1, pp. 532-533] shows that in these cases $\mathfrak{g}$ is of Hermitian type.

Now let $\Sigma$ be nonreduced, i.e., of type $B C_{l}(l \geq 2)$ or $A_{1}$.

Now the discussion after (3.5) and the requirement dim $\mathfrak{g}_{\lambda}=1$ imply, by the table in [H1, pp. 532-533], that $S$ cannot contain any simple root. The union of the $W$-orbits of the simple roots in $\Sigma$ is everything, except the set of longest roots and they form a single $W$-orbit. If $\beta$ denotes the unique shortest simple root, this is the $W$-orbit of $2 \beta$.

Consequently, in the nonreduced case $S$ may only contain the orbit of longest roots. In the non-Hermitian case, again by checking the table in [H1, pp. 532-533], one can see that $\operatorname{dim} \mathfrak{g}_{2 \beta}>1$, showing that in this case $S=\emptyset$. This proves (3.1).

When $\mathfrak{g}$ is of Hermitian type, $I$ is in the center of $\operatorname{Ad}(K)$, whence it follows that $p^{M}$ contains $\mathfrak{a} \oplus I \mathfrak{a}$. To see that $p^{M}$ cannot be larger we observe that, by the discussion above, $S$ may contain only the longest roots of the root systems $C_{l}$, or $B C_{l}$ or $A_{1}$, with the roots having multiplicity one. The number of longest positive roots in these systems is $l$ (resp. 1). So $\operatorname{dim} p^{M}$ cannot exceed $2 \operatorname{dim} \mathfrak{a}$, proving $(3.2)$.

Armed with the result of Lemma 3.1 we can now prove Theorem 0.3. Let $F$ : $\mathfrak{p} \rightarrow \mathfrak{p}$ be a $K$-equivariant map. Then $F$ is, in particular, $M$-equivariant and thus maps $p^{M}$ into itself.

If $\mathfrak{g}$ is not of Hermitian type, (3.1) implies that $F$ is radial.

If $\mathfrak{g}$ is of Hermitian type, then $I=\operatorname{Ad}\left(k_{0}\right)$, where $k_{0}$ is in the center of $K$. In particular, $I$ as a map, $\mathfrak{p} \rightarrow \mathfrak{p}$ is $K$-equivariant and linear.

Hence for any two $K$-equivariant radial maps $F_{j}$, the sum $F_{1}+I F_{2}$ is also $K$ equivariant and its smoothness agrees with that of $F_{j}$.

Now let $F: \mathfrak{p} \rightarrow \mathfrak{p}$ be an arbitrary $K$-equivariant polynomial (resp. $C^{\infty}$ or $C^{\omega}$ ) map. The restriction of $F$ to $\mathfrak{a}$ determines $F$ completely. In light of (3.2) this restriction maps into $\mathfrak{a} \oplus I \mathfrak{a}$. Therefore, it is of the form $f_{1}+I f_{2}$, where $f_{j}: \mathfrak{a} \rightarrow \mathfrak{a}$.

Since $F$ is $K$-equivariant, in particular, it is $M^{\prime}$-equivariant as well. But $k_{0}$ is central in $K$. This yields that $f_{j}$ are $M^{\prime}$-, and consequently $W$-equivariant maps. Now from Theorem 0.1 we know that $f_{j}$ extends to a $K$-equivariant map $F_{j}: \mathfrak{p} \rightarrow \mathfrak{p}$. The maps $G=F_{1}+I F_{2}$ and $F$ are $K$-equivariant and their restriction to $\mathfrak{a}$ is the same. Therefore, $G \equiv F$.

Since a $K$-invariant 1 -form $\alpha$ is horizontal iff the corresponding $K$-equivariant map $h_{\alpha}$ is radial, as an immediate corollary of Theorem 0.3 we get:

Corollary 3.2. Suppose $\alpha$ is a $K$-invariant polynomial (resp. $C^{\infty}$ or $C^{\omega}$ ) 1-form. If $\mathfrak{g}$ is not of Hermitian type, then $\alpha$ is horizontal. If $\mathfrak{g}$ is of Hermitian type, then there exist unique $K$-invariant horizontal polynomial (resp. $C^{\infty}$ or $C^{\omega}$ ) 1-forms $\beta_{j}, j=1,2$, such that $\alpha=\beta_{1}+I \beta_{2}$ and every 1 -form of this type is $K$-invariant. 


\section{ACKNOWLEDGEMENT}

We thank the referee for his question about radial equivariant maps that led to Theorem 0.3.

\section{REFERENCES}

[B] Bourbaki, N., Groupes et algèbres de Lie, Chapitres 4, 5 et 6, Hermann, Paris 1968. MR0240238 (39:1590)

[C] Chevalley, C., Invariants of finite groups generated by reflections, Amer. J. Math 77 (1955), 778-782. MR0072877(17:345d)

[D-Sz] Dancer, A. and Szőke, R., Symmetric spaces, adapted complex structures and hyperkähler structures, Quart. J. Math. Oxford 48 (1997), 27-38. MR1439696 (98e:53083)

[D] Dadok, J., On the $C^{\infty}$ Chevalley's theorem, Adv. in Math 44 (1982), 121-131. MR0658537 (83m:53073)

[H1] Helgason, S., Differential Geometry, Lie Groups, and Symmetric Spaces, AMS, 2001. MR1834454 (2002b:53081)

[H2] Groups and Geometric Analysis, AMS, 2000. MR1790156 (2001h:22001)

[HC] Harish-Chandra, Spherical functions on a semisimple Lie group. I., Amer. J. Math 80 (1958), 241-310. MR0094407 (20:925)

[L] Luna, D., Fonctions différentiables invariantes sous l'opération d'un groupe réductif, Ann. Inst. Fourier, Grenoble 26 (1976), 33-49. MR0423398 (54:11377)

[M1] Michor, P., Basic differential forms for actions of Lie groups, Proc. AMS 124 (1996), 1633-1642. MR1307550 (96g:57041)

[M2] , Basic differential forms for actions of Lie groups. II, Proc. AMS 125 (1997), 2175-2177. MR1401750 (97k:57046)

[Sch] Schwarz, G. W., Smooth functions invariant under the action of a compact Lie group, Topology 14 (1975), 63-68. MR0370643 (51:6870)

[S] Solomon, L., Invariants of finite reflection groups, Nagoya Math. J. 22 (1963), 57-64. MR0154929(27:4872)

Department of Mathematics, lehman College, The City University of New York, Bedford Park Boulevard West, Bronx, New York 10468

E-mail address: adam.koranyi@lehman.cuny.edu

Department of Analysis, Eötvös University, PÁzmány Péter sétány 1/C, Budapest, 1117 HUNGARY

E-mail address: rszoke@cs.elte.hu 